\title{
Extended Exam Wrappers: A Comparison of Approaches in a Learning Strategies Course
}

\section{Ms. Abigail T Stephan, Clemson University}

Abby is a doctoral student in the Learning Sciences program at Clemson University. Broadly, her research interests include intergenerational learning in informal settings, self-directed learning, and cultural influences on the learning process. Abby currently works as a graduate assistant for the General Engineering Learning Community (GELC), a program that supports first-year engineering students in their development of self-regulation and time management skills, effective learning strategies, and positive habits of mind.

\section{Dr. Elizabeth Anne Stephan, Clemson University}

Dr. Elizabeth Stephan is the Director of Academics for the General Engineering Program at Clemson University. She holds a B.S. and a Ph.D. in Chemical Engineering from the University of Akron. Since 2002, she has taught, developed, and and now coordinates the first-year curriculum. As the lead author of the "Thinking Like an Engineer" textbook, currently in its 4th edition, she has been the primary author team-member in charge of the development of the MyEngineeringLab system.

\section{Matthew K. Miller, Clemson University}

Matt Miller is a Senior Lecturer in the General Engineering Program at Clemson University. His background in industrial engineering and engineering education have helped him develop innovative ways to deliver engineering curriculum for freshman engineers and talented high school students interested in engineering. 


\title{
Extended Exam Wrappers: A Comparison of Approaches in a Learning Strategies Course
}

\begin{abstract}
This Complete Evidence-Based Practice paper explores the use of exam wrappers in a learning strategies course designed for first-year engineering students in the General Engineering Learning Community (GELC) at Clemson University. Exam wrappers are most commonly used as tools to facilitate the process of self-evaluation as students reflect on their preparation and performance on a formal assessment, such as a quiz or exam [1]. The learning strategies course accompanying the GELC at Clemson University includes an innovative, extended use of exam wrappers. Currently in its second iteration, the exam wrapper activity is well-integrated into the course and emphasizes the professional significance of self-evaluation and critical reflection in the learning process. Slight modifications to the exam wrapper activity were made between its first [2] and second implementations, and the similarities and differences in outcomes as a result of these modifications will be the focus of the current paper.
\end{abstract}

In the series of exam wrapper assignments, students are asked to complete (1) a reflection detailing what they wanted to happen on their first round of exams, (2) a traditional exam wrapper activity recounting their preparatory behaviors and learning strategies and the results of their exams, and (3) a reflection on what was learned from the experience, and (4) a plan for improvement for each of their STEM courses. This process is repeated following the second round of exams.

One modification of the exam wrapper between its first and second implementation was the introduction of the after-action review terminology. In order to emphasize the students' professional development as engineers, the experience with exam wrappers was framed as a four-stage after-action review. After-action reviews are used to debrief the process and performance on a training event and include four phases: planning, preparation, execution, and follow-up [3]. The exam wrapper activity was intentionally created to model the structure and process of the after-action reviews utilized in industry with the goal of engaging students with the value of gaining real-world skills.

The current study investigates two primary research questions utilizing a qualitative methodological approach. First, it seeks to determine the impact of the exam wrapper activity on subsequent exam scores for students in the second cohort. Second, it aims to understand how the experience with exam wrappers varied between students in the first cohort and the second cohort. The results of the analysis suggest that qualitative themes related to students' experiences differed as a result of the structure and framing of the activity from one iteration to the next. A discussion of the results and implications for educators are provided.

\section{Introduction}

This Complete Evidence-Based Practice paper explores the use of exam wrappers in a learning strategies course designed for first-year engineering students in the General Engineering 
Learning Community (GELC) at Clemson University. Exam wrappers are most commonly used as tools to facilitate the process of self-evaluation as students reflect on their preparation and performance on a formal assessment, such as a quiz or exam [1]. While the structure of exam wrappers varies by discipline, grade level, and instructor preferences, the objective of employing exam wrappers is typically related to enhancing students' metacognitive awareness, or one's ability to effectively plan for, evaluate, and reflect on their learning [1]. Scaffolded activities and experiences that bolster metacognitive awareness are increasingly significant for contemporary engineering students as they prepare for the problem-solving demands and self-directed nature of an evolving, dynamic industry. A student's ability to be aware of what and how they are learning enables them to become more self-directed and self-regulated in their learning; this in turn will help students develop the skills to be more productive and innovative as they continue their work as students and engineering professionals.

The current paper will begin by describing the traditional use and purpose of exam wrappers in higher education, followed by the unique design and purpose of modified exam wrapper activities within the learning strategies course for students in the GELC at Clemson University. This section will include the specific processes utilized in the first and second iterations of the activity. Then, the methods used to analyze the exam wrapper data will be explained, followed by results. Finally, a discussion outlining the meaning of the results and implications for practitioners will be provided.

\section{Exam Wrappers}

Exams wrappers are often used as a tool to scaffold students' evaluation of their preparation and performance on graded assignments, including projects, quizzes, and exams. An exam wrapper is typically a worksheet or form created by instructors asking students a series of questions related to their performance on an assignment and prompting students to consider why they lost points on specific questions or sections [1].

Within the scope of higher education, exam wrappers are popular in STEM courses as they aid students in both assessing and reflecting on their learning [4], [5], as well as refining their study strategies [6]. Additionally, a benefit of using exam wrappers is that they effectively promote metacognitive awareness [1] by prompting students to think about their behaviors and thought processes. Through exam wrappers show considerable promise in practice, empirical evidence of wide range use of this self-evaluative tool is not abundant in the literature. Some specific collegiate courses that have documented their use of exam wrappers include engineering mechanics [4], food science and human nutrition [5], introductory science [6], as well as introductory calculus, statistics, and chemistry [1].

\section{Modified Exam Wrapper}

In order to meet the needs of our students, a modified exam wrapper assignment has been implemented in the learning strategies course associated with the GELC. The first iteration, detailed in our 2019 ASEE paper [2], took place in Fall 2018. The second iteration, which will be the primary focus of the current paper, took place in Fall 2019. For the purposes of this paper, 
the students enrolled in the learning strategies course during the Fall 2018 semester will be referred to as the first cohort, and the students enrolled in the learning strategies course during the Fall 2019 semester will be referred to as the second cohort. The following section will detail the structure and purpose of the exam wrapper activities in each of the two cohorts.

\section{First Cohort (Fall 2018)}

The exam wrapper activities for the first cohort, which consisted of 91 first-year engineering students, included an initial reflection prior to the first round of exams, a traditional exam wrapper after the first round of exams focused on behaviors and knowledge of course learning outcomes, selection of an evidence-based learning strategy to incorporate between the first and second round of exams, and a traditional exam wrapper after the second round of exams.

The first cohort's experience with exam wrappers is detailed in our 2019 ASEE paper [2] and included three phases: before the first round of exams, following the first round of exams, and immediately after the second round of exams. Students were given the option to complete the exam wrapper activity for one of three STEM courses: chemistry, math, or engineering. Students were encouraged to select either the course in which they received the lowest first exam grade or the course in which they felt least comfortable with the material. In addition to articulating their experiences with a growth mindset, students achieved higher exam scores after completing the exam wrapper.

\section{Second Cohort (Fall 2019)}

The second cohort consisted of 76 students. Their experience with exam wrappers was framed as a four-stage after-action review in order to correlate their experiences as students and their future professional careers as engineers (Appendix A). After-action reviews, originating from the military, are used to debrief the process and performance on an event or activity and include four phases: planning, preparation, execution, and follow-up [3]. Often used as informal evaluations of a team's performance, after-action reviews should be framed as an opportunity for growth [7]. In industry, these reviews are sometimes referred to as "post mortems" or "lessons learned," with the main idea being to provide a systematic way to capture process improvement [8]. The exam wrapper activity was intentionally created to model the structure and process of the after-action reviews utilized in industry.

An additional modification to the exam wrapper activity in the second iteration included the integration of the Skillful Learning categories of metacognition [9]. In the second stage of the exam wrapper after-action review, students completed a form detailing their study behaviors within each of the three categories: rehearsal, elaboration, and organization. In the third and fourth stages, students reflected on their use of strategies within each category and developed an improvement plan for bolstering their involvement in each category. Explicitly threading metacognitive concepts into the exam wrapper activity highlights the importance of metacognition. 


\section{Research Questions}

To gain a deeper understanding of the effects of the exam wrapper activity utilized by the GELC at Clemson University, we pose the following questions:

1. In what ways does framing the exam wrapper activity as an after-action review influence the perceived utility of exam wrappers in first-year engineering students in the second cohort?

2. How does the impact of the exam wrapper activity vary for students in the first and second cohorts?

\section{Methods}

The current study utilizes a qualitative methodological approach, including a comparative case study [10], to answer our research questions. Comparative case studies draw out similarities and differences between two or more sets to identity similarities and differences and determine effective practices or policies [10]. In this study, we have two cases: the first cohort and the second cohort. The comparison will focus on how similarities and differences in the two approaches impact broad student outcomes. Since new data related to the first cohort was not collected or analyzed in this study (only compared to the results of the analysis for the second cohort), information related to sample, data collection, and data analysis for the first cohort is not included below. For this information, please refer to our 2019 ASEE paper [2].

\section{Sample}

The sample included students enrolled in each of the four sections of the learning strategies course associated with the GELC who provided consent for their work to be used and completed all components of the exam wrapper assignment. Additionally, to be eligible for participation in the current study, students needed to complete all formal exams or assessments linked to the exam wrapper activities. These qualifications provided us with a total sample of 54 students, or $71.05 \%$, from the second cohort, compared to 78 students, or $85.71 \%$, from the first cohort.

\section{Data Collection}

The specific pieces of student data collected for this study include all components of the exam wrapper activity. These materials include several assignments associated with the first round of exams (Exam Wrapper After-Action Review \#1 Stage 1 [Appendix B], Stage 2 [Appendix C], and Stage 3\&4 [Appendix D]), as well as the assignment associated with the second round of exams (Exam Wrapper After-Action Review \#2 Stage 2 and Stage 3\&4). Stages 3 and 4 were combined into one assignment to ensure the reflection on preparation and performance was closely tied to goals going forward. Stage 1 was not repeated in After-Action Review \#2 since students had documented what they wanted to happen on their second round of exams in Stage 4 of the After-Action Review \#1. 


\section{Data Analysis}

Open-response reflection data collected from the exam wrapper activity materials of students in the second cohort were coded thematically to reveal common, relevant student experiences. Each component of the exam wrapper assignment was read in its entirety to identify emergent themes between and across students in the second cohort. As student responses were read, specific quotes highlighting student experiences were gathered. Student quotes included in this paper are de-identified, and all names attached to the quotes are pseudonyms. Due to time restrictions, only one author coded responses and created themes. Several instructors of the course reviewed the themes, along with student quotes to support the creation of each theme, in an attempt to validate the results.

To determine how the first cohort's experience compared to the second cohort's experience, a comparative analysis was conducted. More specifically, a comparison of themes that emerged from the first and second cohorts' exam wrapper assignments was conducted. The results, sorted by research question and portrayed as emergent themes, are presented below.

\section{Results}

Research Question 1: Second Cohort Students' Perceived Utility of Exam Wrapper as an AfterAction Review

Broad themes of the exam wrapper activity for the second cohort of students include awareness of changes that need to be implemented in future iterations, growth mindset/willingness to learn how to learn, and the importance of preparation. General observations and representative student quotes related to each of these themes are presented below.

Theme \#1: Awareness of Changes That Need to be Implemented

After the first round of exams, students became increasingly aware of the challenges that they faced before and during their exams. For example, students highlighted weaknesses in their preparation and test-taking skills, followed by modifications that they plan to implement.

Though aware of weaknesses in their preparation and test-taking skills, some students discussed being unsure of how to make changes to their preparation following the first round of exams. For example, one student stated that they "underestimated the idea of studying." Some students described feeling unprepared when it came to solving problems but failed to follow-up with future actions to take. However, many of these students were able to create concrete actions steps going forward to fulfill their goals during the second round of the exam wrapper.

The student quotes presented below exemplify this theme:

"Next time however, I want to start studying sooner in the week in shorter increments to truly learn the material" (Aaron). 
"The biggest change for me in college isn't the severity of the classes, it's the time management” (Becca).

"My biggest problem is I run out of time on the tests and aren't [sic] able to finish all the problems” (Steve).

"There were several problems where I had been using the 'problem matiching' [sic] mentioned in the 'Reflecting on your Thinking' video, so when it came time for the test and the problem wasn't what I had practiced, I couldn't complete the problem. I also made many tiny errors because I hadn't been focusing on all of the concepts, only the concepts I thought I would need to know" (Becca).

"I have carried over some of my studying habits that worked for me in high school, but have definitely had to make changes" (McKayla).

"In the future I realize that I need to spend more time with the material so I will have a better understanding of it” (Sam).

Theme \#2: Growth Mindset/Willingness to Learn

Many students expressed a positive, open attitude towards improving their performance. After recognizing the challenges ahead of them, they reframed their future learning as an opportunity for growth. The following student quotes illustrate this attitude:

"As the year goes on I will learn more and more about ways to prepare and study for my exams" (Baron).

"Now that I am in college I have to actually learn how to study and learn which study methods work best for me. I am currently thinking about going back to my dorm and watching different TED Talks or YouTube videos about studying to see which method I think would work best for me. I realized for math that I need to do practice problems each day, however, I still haven't found out what works best for me when it comes to studying actual notes. Office hours and the practice test definitely helped me with studying also" (Malik).

"Now I feel like I have to teach myself and train myself essentially how to study again" (Rhoda).

"I have to make choices and balance my life with studying, homework, social things and all sorts of other stuff so I think that adds a whole new level of challenge to this" (Holly).

Theme \#3: Importance of Preparation

Students were clear about the actions that they took to prepare for their exams. Some of these actions include utilizing course support services, attending professors' office hours, working 
practice problems, and completing practice exams. Some students were also able to articulate a keen awareness of the importance of preparation for their performance on exams.

"I studied weeks in advanced [sic] and made sure that I studied for each exam every night leading up to the exams and that I got help when I didn't understand something" (Kelia).

"I created a plan for myself. I made my studying more consistent and made myself understand the topic not just memorize it" (Connor).

"I went to a lot of the study times and the reviews for the exam, I also did some on my own, so I felt really prepared for that exam" (Lucas).

"Going to office hours also helped me better understand what we were learning in class, especially for matrices. I also went to the [academic success center] and did a practice quiz which was just like the actual exam" (Malik).

"I felt that I was prepared for the exams, because I studied and went to tutoring, office hours, and exam prep sessions for help. I knew my material. I worked many problems and ask my classmates and friends questions" (Henry).

"I did feel prepared for my assessment, because I read the book, reviewed practice problems, and watched study videos on the topics I was about to take assessments on" (Carrie).

Research Question 2: Comparison of Exam Wrapper Experience for First and Second Cohorts

Themes from the first cohort included: 1) increased preparation, 2) enhanced confidence, 3) recognition of insufficient study practices, and 4) increased understanding of oneself as a learner. To reiterate, themes from the second cohort include awareness of changes that need to be implemented in future iterations, growth mindset/willingness to learn how to learn, and the importance of preparation.

In comparison to the second cohort, the first cohort articulated a greater range of experiences, as evidenced by the quantity of themes. One of the most salient themes from the first cohort that did not transfer to the second cohort was enhanced confidence. Many students in the first cohort, in addition to feeling more prepared, discussed a sense of competence and confidence going into their second round of exams. Though students in the second cohort acknowledged the importance of preparation in achieving desired exam scores, they did not expressly state an increase in confidence levels.

Students in the second cohort spoke about their experience more objectively than students in the first cohort. Most of the students were able to discuss what happened, identify their weaknesses, and create a plan for improvement going forward without being overly critical of themselves 
personally. In contrast, students in the first cohort often expressed feeling a sense of personal failure.

\section{Discussion}

The comparative case study revealed critical differences between students in the first and second cohorts. Most notably, students in the second cohort were able to speak of their experience more objectively and analytically. This was likely due in part to the emphasis on professional development and after-action reviews. Students viewed this experience as an opportunity to troubleshoot aspects of their preparation not going as well as anticipated. Accordingly, adjustments were perceived as necessary for bolstering performance, not as a personal attack. This finding reflects the adapted framing and formatting of the exam wrapper activities presented to the second cohort. Though preliminary, it may suggest that taking a professional approach and utilizing the language of after-action reviews bolsters students' ability to accurately self-evaluate, create an actionable plan, and make adjustments.

A limitation of the current study relates to the sample. The sample drawn from both the first and second cohorts is comprised of students who completed all required components of the assignment. This suggests that students included in our sample were more likely to be high academic achievers and more concerned about their performance on assessments. Furthermore, the series of activities were due at several checkpoints throughout the semester, suggesting that the students included in the study were consistently engaged academically. In future work, our program should conduct follow-up interviews with students who did turn in all assignments as well as those who did not to better understand potential profile differences between the students included in and excluded from the current sample.

Along with the structure of the exam wrapper activities, the structure of the programming differed between the Fall 2018 and Fall 2019 cohorts' experiences. For example, in Fall 2018, the learning strategies course was taught by various university personnel, including a senior engineering faculty member, an associate dean of the university, a course support programs coordinator, and a graduate student. In contrast, during the Fall 2019 semester, all four sections of the learning strategies course were taught by engineering faculty. Additionally, the students in the second cohort, Fall 2019, were enrolled in an alternate curriculum path from the first cohort. These external differences between the two samples make it difficult to form conclusive statements around the efficacy of the modified exam wrapper format. There will be a continuation of the Fall 2019 curriculum path for the Fall 2020 cohort. Comparisons between groups with the same curriculum will be made in future work.

Finally, while this paper focuses on the short-term impact of the exam wrapper activity, future research should consider the long-term effects of the exam wrapper activity on student outcomes, such as retention and grade point average. The long-term impact of this self-evaluative tool for students is not well-documented in the literature. Developing an understanding of the exam wrapper activities that "stick" with students throughout their academic studies could provide guidance for future iterations of this assignment. 


\section{Conclusion}

The purpose of this complete research paper was to investigate the efficacy of an iterative course assignment, exam wrappers, and explore differences between two cohorts' experiences. Results of this complete research paper suggest that, while both positive, the experiences of the two cohorts were unique. Due to modifications in the framing of the exam wrapper activity, students in the second cohort viewed the exam wrapper as an opportunity for professional growth. Based on these results, engineering educators should consider infusing language related to professional development and industry practices within their assignments.

\section{References}

[1] B.G. Trogden, and J.E. Royal, "Using exam wrappers in chemistry and mathematics coursework to encourage student metacognition," Journal on Excellence in College Teaching, [in press 2019].

[2] A. Stephan, L. Whisler, E.A. Stephan, and B. Trogden, "Using exam wrappers in a selfdirected first-year learning strategies course," in ASEE Annual Conference and Exposition 2019, Tampa, Florida, USA, June 2019.

[3] M. Smith, "Why after-action reviews are so important: Military strategy could help you take your organization to the next level," Michael Hyatt, August 2018. [Online]. Available: https://michaelhyatt.com/why-after-action-reviews-are-so-important/ K. Elissa, "Title of paper if known," unpublished.

[4] A. Badir, J. Liao, T. Kunberger, G.I. Papkov, L.D. Nguyen, and R. O’Neill, "Exam wrappers, reflection, and student performance in engineering mechanics," in ASEE Annual Conference and Exposition 2018, Salt Lake City, Utah, USA, June, 2018.

[5] P. Gezer-Templeton, E. Mayhew, D. Korte, and S. Schmidt, "Use of exam wrappers to enhance students' metacognitive skills in a large introductory food science and human nutrition course," Journal Of Food Science Education, vol 16, no. 1, pp. 28-36, 2017.

[6] K.J. Metzger, B.A. Smith, E. Brown, and P.A.G. Soneral, "SMASH: A diagnostic tool to monitor student metacognition, affect, and study habits in an undergraduate science course," Journal of College Science Teaching, vol 47, no. 3, pp. 88-99, 2018.

[7] M. Darling, C. Parry, and J. Moore, "Learning in the thick of it," Harvard Business Review, July-August 2005. [Online.] Available: https://hbr.org/2005/07/learning-in-the-thick-of-it.

[8] B. Collier, T. DeMarco, and P. Fearey, "A defined process for project post mortem review," IEEE Software, vol. 13, no. 4, pp. 65-72, July 1996.

[9] P. J. Cunningham, H. M. Matusovich, C. Venters, S. A. Williams, and S. Bhaduri, "Teaching metacognition: Helping students own and improve their learning," American Society for Engineering Education Annual Conference and Exposition, Salt Lake City, UT, USA, 2018. Workbook available from https://skillful-learning.org/wp-content/uploads/2018/06/2018-

ASEE-Metacognition-Workshop-Workbook.pdf

[10] S.J. Fox-Wolfgramm, "Towards developing a methodology for doing qualitative research: The dynamic-comparative case study method," Scandinavian Journal of Management, vol. 13, no. 4, pp. 439-455, December 1997. 


\section{Appendix A: After-Action Review Assignment Framing}

The following purpose and requirement statements are included at the top of each exam wrapper assignment.

Purpose: Originally developed by the US Army, an After Action Review (AAR) is a formal process used to analyzing what happened, why it happened, and how it can be done better. This method can have various names, but almost all companies now have some form of this review to evaluate an event in their business and how to use those lessons learned to move forward. In this assignment, you will conduct an AAR of one of your STEM-class exams to examine what happened, why it happened, and how you can do things better for the next exam.

Requirements: There are four stages to an AAR. Complete an AAR regarding the results of your most recent assessment in one of your STEM courses (Chemistry, Engineering, Math). This portion of the assignment is STAGE [X].

\section{Appendix B: After-Action Review \#1 Stage 1 Prompt}

Stage 1: What you wanted to happen

Copy the following question prompt into the text box, and answer the questions.

Think back to the start of the fall semester, at early move-in or the first week of classes.

- How did you see the semester going academically? How did you think you would perform on your examinations?

- How much different from high school did you anticipate studying in college would actually be?

Now think back before you took the examinations.

- Did you feel prepared for the assessments? 


\section{Appendix C: After-Action Review \#1 Stage 2 Prompt}

Stage 2: What actually happened

- Obtain the results of your recent assessments for Chemistry, Engineering, and/or your Math classes. Do not complete the Google Form until you have these results for all your STEM classes available to you.

- Complete the Google Form [link here].

- The results will be sent to you via email. Save the email as a PDF file named AAR1_Stage2_username, where username is the first part of your Clemson email address.

- Upload the PDF to the Exam Wrapper | After-Action Review \#1: AAR1_Stage2 [assignment link here].

The Stage 2 results will be used to complete Stage 3: Learn from the experience and Stage 4: Adjust your behavior. These stages are discussed in the Exam Wrapper | After-Action Review \#1: AAR1_Stage3and4 assignment [assignment link here].

Google Form Introduction Page

\section{Exam Wrapper After-Action Review I Stage 2}

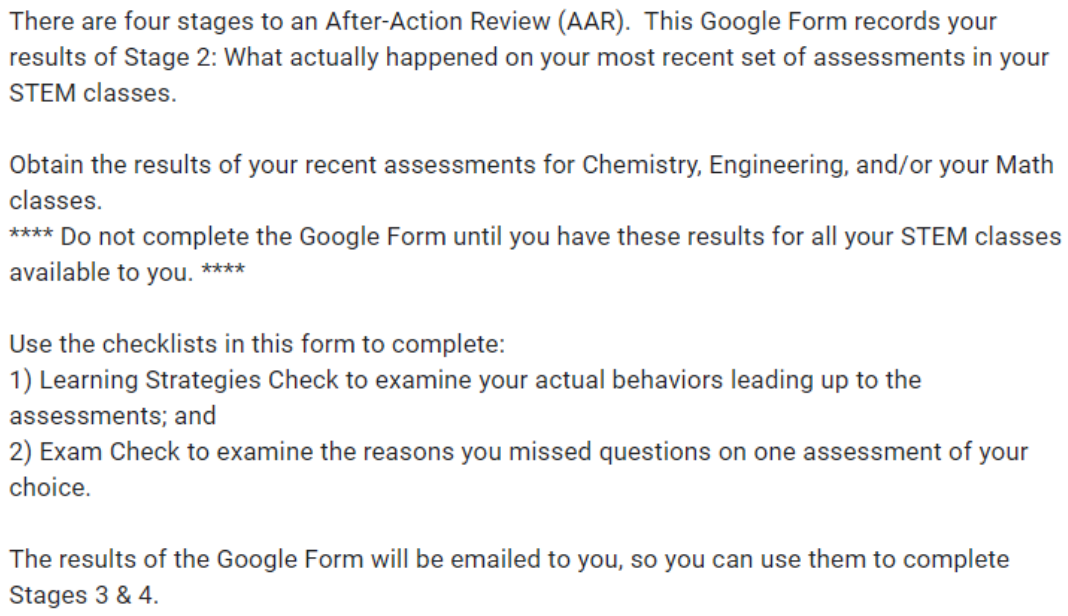

To view all seven sections of the Google Form, email AUTHOR at EMAIL for access. 


\section{Appendix D: After-Action Review \#1 Stage3\&4 Prompt}

Stage 3: Learn from the experience

- Based on your responses to Stage 2, do you spend more of your time and effort with rehearsal, elaboration, or organization strategies while studying?

- Identify one strategy from each area that you could add to improve effectiveness in your learning. Discuss how you think each strategy could help you.

○ Rehearsal

- Elaboration

- Organization

- For missed points identified on the Google Form in Stage 2, provide the following information to identify the learning outcomes that you need to go back and re-learn. You may need to visit your instructor during office hours in order to see the exam and answer this question. Include each question that was not $100 \%$ correct on your assessment. Only use as many of the lines are needed for your assessment.

\begin{tabular}{|l|l|l|l|}
\hline Question \# & Learning Outcome & Textbook Pages & Reason \\
\hline & & & \\
\hline & & & \\
\hline & & & \\
\hline
\end{tabular}

Stage 4: Adjust your behavior

If you don't apply what you learned, things will not improve. Think about what behaviors you need to change before the next assessment to be successful.

- You currently are using a professional strategy for your learning journal. Compare the benefits of that professional strategy against the needs you identified here for increased effectiveness.

- Discuss whether keeping that strategy or adopting a specific new strategy will help you be most effective.

- If adopting a new strategy, identify it here and explain how you think it will help you. 